\title{
Multimedia Synchronization for Handoff Control with MPEG in All-IP Mobile Networks
}

\author{
Gi-Sung Lee ${ }^{1}$, Hong-jin Kim ${ }^{2}$, Il-Sun Hwang ${ }^{3}$ \\ ${ }^{1}$ Dept. of Computer Science Howon Univ., Korea \\ ygslee@sunny.howon.ac.kr \\ ${ }^{2}$ Dept. of Computer Information, KyungWon College, Korea \\ ${ }^{3}$ R\&D Network Management, KISTI, Korea
}

\begin{abstract}
This paper proposes a handoff management scheme for the synchronization algorithm of an all-IP based multimedia system. The synchronization algorithm and handoff management method are proposed to realize smooth play-out of a multimedia stream with minimum loss under handoff conditions which normally occur due to the movement of mobile hosts. Handoffs, which frequently occur under mobile environments, result in loss of multimedia streams stored in base stations due to the change of base station. As a result, the multimedia stream shows a low QoS due to disruption of the stream at play-out. The proposed scheme shows that it not only provides a stream of continuous play-out but also shows a higher packet play-out rate and lower loss rate than previous methods.
\end{abstract}

\section{Introduction}

The recent explosive increase in Internet utilization has accelerated the emergence of new services based on the mobile computing environment. As a result, the existing service architecture, which is based on simple communication between the client and the server, is no longer capable of satisfying the needs of users who want to utilize various types of high-speed multimedia services $[1,2]$. This limitation of existing communication service architecture can be overcome by expanding the concept of service provision from one that is single system based to a mobile system connected by a wireless network: the mobile environment provides key functionalities which enable such performance expansion. In a mobile environment, play-out of multimedia data on a mobile host is difficult due to inherent characteristics in wireless networks such as the high data loss rate, long delays and low network bandwidth. The distributed multimedia systems which are connected to the wireless network in large numbers use buffers to overcome network delays and unpredictable losses.

A Base Station (BS) transmits Group of Picture (GoPs) of MPEG from multiple multimedia servers. However, if the expected play-out time is faster than the arrival time, unpredicted delays and increase in traffic can cause failures in play-out of subframes. To solve this problem, buffering is needed at BS in order to reduce interpacket jitter delay between the multimedia server and BS in a mobile environment. A mobile network offers the advantage that Mobile Hosts $(\mathrm{MH})$ can move within the network 
$[3,4,6]$. However, the disadvantage is that multimedia streams transmitted to a BS must be retransmitted. The disadvantage of handoff is that the MPEG video data stored in a BS buffer can be lost. In this paper, the BS and the MH were configured with a 2-jitter buffer and 1-jitter buffer, respectively. In addition, for the GOP (I, P, B) of the MPEG video generated and lost during handoff, the I-picture of the MPEG video of the old BS is transmitted to the new BS. The proposed method aims to enable play-out of the substream stored in the buffer of new BS without retransmission so that adverse effects on play-out that result from loss of media are prevented.

\section{Related Work}

Current research has reached a level, in which synchronization schemes based on wireless communications are incorporated into conventional ones. D. K. Y. Yau and S.S. Lam adopted a frame rate adjustment scheme in which the CPU processing time of a video server is adjusted according to the frame transmission rate, and traffic load on network links is monitored using kernel-level threads. This leads to a reduction in network traffic by increasing or decreasing frame transmission rates. However, one drawback of this approach is that control is performed only on the server side [4]. M. Woo, N. U. Qazi, and A. Ghafoor defined a BS as an interface between wired/wireless networks. For wired networks, the interface defines buffering in the BS to reduce jitter delay between packets. One possible shortcoming of this approach is an attempt to apply buffering to synchronization by assigning existing wireless communication channels [5].

Azzedine Boukerche proposed an efficient distributed synchronization problem in wireless and mobile multimedia systems to ensure and facilitate mobile client access to multimedia objects [1]. He also proposed synchronization and handoff management schemes that allow mobile hosts to receive time-dependant multimedia streams without delivery interruption while moving from one cell to another [2]. But in this method, delay and overhead due to forwarding of MMU data from the buffer of an old BS to a new BS adversely affects the play-out of MMU in MH.

\section{Multimedia Synchronization for Handoff in MPEG GOP}

\subsection{System Architecture}

This system supports $\mathrm{k}$ multimedia server nodes, $\mathrm{m} \mathrm{BS}$, and $\mathrm{n}$ MHs. The BS communicates with ith MH in the mth cell. These MHs access the server via the BS. This system monitors variations in the start time for transmission as well as buffers at the BS, using variables such as arrival time of subframes transmitted from multimedia servers and delay jitter. Some advantages of this system include its capability to overcome the limitations of mobile communications like small memory size and low bandwidths. The MPEG video data stored in the multimedia server are composed of data streams split into $G O P_{k}^{i}$ units in the sequence, based on a synchronization group, 
not on the same byte. Figure 1 shows the configuration for the proposed system. Handoff refers to a state in which a MH moves out of range of a BS and comes into range of another. If handoff takes place during MPEG video transmission, data being transmitted from the BS buffer are lost. This system has architecture which passes only I-frames specific to MPEG videos into buffer of new BS for the transmission and the play-out. Figure 1 shows an overview of system modules for synchronization of MPEG videos in mobile environments. MPEG video streams are retrieved from a frame of a certain type stored within the database in the multimedia server, and the sender transmits them to the $\mathrm{MH}$ via the $\mathrm{BS}$. The $\mathrm{MH}$ receives bit streams in a frame of a certain type from the multimedia server. The frames stored in the buffer are converted by the MPEG decoder, and are then played out.

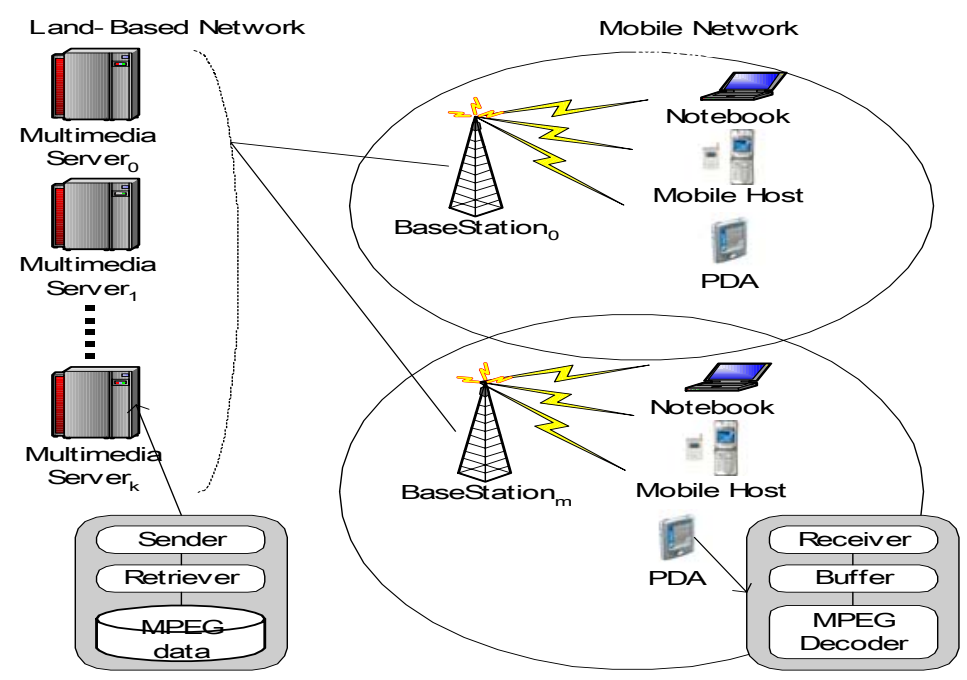

Fig. 1. Synchronization System for MPEG Video

\subsection{Control of Multimedia Server's Transfer Rate}

There are MPEG frame in 3 types: I, P and B frames. During the decoding process, I and $\mathrm{P}$ frames are considered more important than B frames. If the GoP loss rate increases due to overload, the server's transfer rate can be reduced by selectively discarding $\mathrm{B}$ frames to reduce the loss rate of I, P frames so that the reception rate of I, P frames increases. Therefore, the picture quality is better than if no transmission rate control is applied on the server. If, at time t, time is insufficient when $t_{d}$ is imminent, $\mathrm{GoP}$ is discarded. That is, if the number of possible transmissions $r_{t}$ is 0 , GoP is discarded. Here, the server's transmission rate is controlled by selective discarding of GoP according to the loss rate of transmitted GoP. Taking this into consideration, a method for controlling the server's transfer rate according to the GOP loss rate within the network has been proposed in figure 2 . 
Procedure Control of Server's Transmission Rate

$\operatorname{If}\left(\rho>={ }_{B l H}\right)\{$

While (wait until message of $B_{B_{\text {nomal }}}$ is received from BS)

Wait $/ /$ decrement

else if $\left(T_{1 L}<\rho<T_{1 H}\right)$

Transmit/maintain $\operatorname{GoP}(I, P, B)_{k}^{i}$ else

While (wait until message of $B_{\text {nomal }}$ is received from BS) transmit $G o P(I)_{k}^{i} / /$ increment

Fig. 2. Control of server's transmission rate according to GoP loss rate

\subsection{Controlling the Play-Out Time of the MH}

In the method proposed by Chen [4], the server's transmission time is reconfigured using feedback information from $\mathrm{MH}$; here, a new transmission time is set. However, this involves a complicated method of setting the new transmission time, and is not suitable for an Mobile environment where there is no guaranteed limit for loss and delay. In selecting a control for delay variation under such a network environment, resetting the estimated play-out time of $\mathrm{MH}$ is more desirable than controlling the server's transmission time. The proposed algorithm for controlling the estimated play-out time according to the delay variation of the synchronization method within the media is illustrated in figure 3. After receiving RTT GoP, packets are separated and a two-stage synchronization process within the media is applied. In the first stage,

Procedure Controlling the estimated play-out time While (not EOF) \{

Get a new GoP; $\mathrm{D}=\mathrm{SCR}-\mathrm{STR}$;

if $\left(\mathrm{D}>\tau_{p}\right)\{$ Insert into decoding buffer; $\}$

If(D $>0)\{$ Insert into Delay buffer; \}

Else $\{$ Discard $;\}$

If $\left(B_{d L}<\bar{D}<B_{d L}\right) / / \bar{D}$ is average delay time continue;

else if $\left(\bar{D}<=B_{d L}\right)\{$

$P_{\tau}=P_{\tau}-\delta ; / / P_{\tau}$ is interval time, $\delta$ is max jitter time

continue; $\}$

Else $\left\{P_{\tau}=P_{\tau}+\delta\right.$

continue; $\}$

Fig. 3. Controlling the estimated play-out time 
the amount of GoP delay occurring within the mobile network is calculated. Since the server transmits packets based on SCR, the received SCR is the time of the server's packet transmission, and the STC of MH at reception point is the packet's arrival time. Therefore, the delay can be calculated in the difference between the received packet's SCR value and the STC value of MH at arrival time. The second stage is the process of reconfiguring the estimated play-out time of $\mathrm{MH}$ according to network delay: it consists of estimated play-out time delay action and estimated play-out time progression action. The average delay $\bar{D}$ is calculated by passing the delay $\mathrm{D}$ experienced by each packet through a low-pass filter

\subsection{Handoff Control}

This paper is intended to enable a MH to implement the play-out of media streams within QoS limits, without any loss of multimedia data or additional delays accompanying message transmission during a handoff. In this paper, the $H M_{i}$ provides multimedia service via the $B S_{m}$. The BS is classified into a primary BS and non-primary BS. The former is a BS responsible for sending MPEG video data in GOP units to the MS, while the latter is a BS adjoining the primary BS.

The buffer size within BS has the two-jitter size, while the one within MH has the one-jitter size. Such buffer configurations offer the advantage of disallowing loss of MPEG video during a handoff, unlike the configuration scheme where the buffer works only within BS. This configuration strategy also offers complementary configurations for MH's small memory size. Handoff occurs when $M H_{i}$ travels out of range of $B S_{\text {current }}$ into the range of $B S_{\text {new }} \cdot B S_{\text {current }}$ reports a handoff message to the multimedia server and starts handoff processing. The algorithm is described as follows.

\section{Algorithm Handoff Control}

1. $M H_{i}$ performs playout for a time equal to (GoP $\left.\times \gamma\right) \times \lambda$ regardless of handoff. Here, $\gamma$ is the play-out speed time required by the substream and $\lambda$ is the maximum jitter time within the media.

2. $M H_{i}$ notifies the Handoff on message indicating handoff has occurred to the multimedia server and $B S_{\text {current }}$.

3. Setting performed: $B S_{\text {old }}=B S_{\text {current }}$ and $B S_{\text {current }}=B S_{\text {new }}$.

4. $B S_{\text {nen }}$ sends message Handoff $f_{\text {on }}$ to multimedia server and notifies that the multimedia server to stops transmission of GOP.

5. Each multimedia server transmits $G O P(I)_{k}^{i}$ to $B S_{\text {new }}$ as soon as it receives Handoff on .

6. Only the $G O P(I)_{k}^{i}$ of the MPEG video which exists in the buffer of $B S_{\text {old }}$ is forwarded to the buffer of $B S_{\text {new }}$. 
7. After it has received $G O P_{k}^{i}(I)$ from the buffer of $B S_{\text {old }}$ through the server, it sends Handoff $_{\text {off }}$ message to the multimedia server and mobile host to notify that handoff has been completed successfully.

8. $M H_{i}$ sends request messages to each server to perform a normal transfer.

\section{Performance Evaluation}

Presented are the simulation experiments we carried out to evaluate the performance of our synchronization scheme. We developed a distributed discrete-event model to simulate a cellar wireless multimedia system. Simulation is performed using IBM compatible PCs with Pentium or an equivalent processor. Interfaces and algorithms were written using the JAVA development kit JDK 1.3, which were stored in Microsoft MDB as simulation.mdb files. For the purpose of this paper, we have assumed that all simulations are performed in mobile environments. In order to ensure proper packet processing, we applied the information used for actual simulations, computed using the Poisson distribution, equally to mobile networks which have 300 channel for 60 cells. One thousand GOP frames were used in performance evaluation experiments to which a maximum delay jitter time of $600 \mathrm{~ms}$ was applied. Table 1 displays the simulation parameters we used in our experiments.

Table 1. Simulation parameters

\begin{tabular}{l|l|l|l|}
\hline $\begin{array}{l}\text { Number of cells } \\
\text { Number of servers }\end{array}$ & 60 & $\begin{array}{l}\text { Forward time to BS } \\
\text { Rate of handoff }\end{array}$ & $\begin{array}{l}100 \mathrm{~ms} \\
5 \% \text { of } \\
\text { MMUs } \\
\text { Buffer size of a MH }\end{array}$ \\
$\begin{array}{l}\text { Buffer size of a BS } \\
\text { 2 Jitter }\end{array}$ & $\begin{array}{l}\text { RTT to request/deliver a GOP } \\
\text { Average Jitter }\end{array}$ \\
Play-out times/GOP & $250 \mathrm{~ms}$ & Maximum Jitter & $600 \mathrm{~ms}$ \\
\hline
\end{tabular}

Figure 5 shows the GoP loss rate of $\mathrm{MH}$. Here we can see that, if control is performed, the number of transmitted GoPs is reduced if the loss rate is increased so that the loss rate is reduced.

Figure 5 shows the loss rate of GOP at MH. Here, the GoP from 15 to 20 seconds is subject to handoff, therefore the buffer data of $B S_{\text {old }}$ is sent to the buffer of $B S_{\text {new }}$ in order to prevent the loss of MPEG video's I-frame as a result of handoff. In addition, for handoff, by delaying $10 \mathrm{~ms}$ of maximum delay jitter $\lambda$ at the I-picture interval time $\tau$ of $G O P_{k}^{i}$ at $M H_{i}$, a method for obtaining more time for moving data at $B S_{\text {old }}$ to the buffer of $B S_{\text {new }}$ has been proposed.

As shown in Figure 5, previous research results failed to deal with handoff and as such suffer from loss of substream data in the buffer of $B S_{\text {old }}$. 


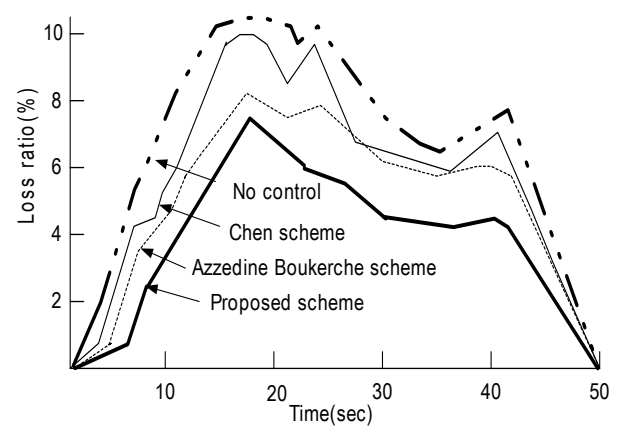

Fig. 5. Comparisons of GoP loss rate in $\mathrm{MH}$

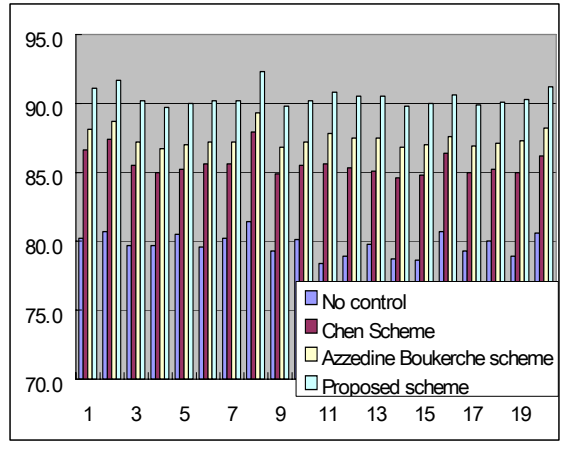

Fig. 6. Comparisons of play-out rate

Figure 6 shows the results of 31 tests in which the arrival time was changed for each experiment. In Test 2 and 8, minimum delay and maximum delay were adjusted to $50 \mathrm{~ms}$ and $600 \mathrm{~ms}$ each; compared with the no control case, the play-out rate was improved by $6 \%$ for the Chen scheme, by $8 \%$ for the Azzedin-Boukerche scheme and by about $10 \%$ for the scheme proposed in this thesis.

In Test 4, 9 and 26, minimum delay and maximum delay were adjusted to $20 \mathrm{~ms}$ and $800 \mathrm{~ms}$, respectively, to induce overflow and starvation. In these cases, while network traffic conditions worsened, the play-out rate was improved by $5 \%$ for the first variance and by $8-9 \%$ for the second variance. In average, the play-out rate was about $79 \%$ for the conventional method, $85 \%$ for the first variance and $91 \%$ for the second variance.

\section{Conclusions}

We proposed a scheme that uses MPEG-frame within GOP in mobile networks. In addition, delay and overhead due to forwarding of MMU data from the buffer of an old BS to a new BS adversely effects play-out of MMU in MH. Therefore, to solve this problem, we propose an algorithm which forwards only the I-frame of MPEGframe to the new BS. The MPEG-frames within the buffer of MH are presented as adding maximum delay jitter to the interval time of GOP because the play-out policy is related to the occupancy rate of the buffer during handoff. Therefore, we propose an algorithm that performs each overflow policy and underflow policy based on buffer watermarking. Moreover, we propose a scheme that not only deals with handoff quickly by controlling the buffer and play-out policy, but also properly handles limiting factors for mobile communications such as small memory size and low bandwidths. The proposed scheme allows the BSs to control handoff and the play-out policy, and further provides a solution to the problems of handoff in mobile networks. After evaluation, it has been shown that the proposed scheme offers continuous MPEG data play-out, higher packet play-out rates, and lower packet loss rates, relative to the conventional scheme. 


\section{Reference}

1. Azzedine Boukerche, Sungbum Hong and Tom Jacob, "MoSync : A Synchronization Scheme for Cellular Wireless and Mobile Multimedia System", Proc. Ninth International Symposium on Modeling, Analysis and Simulation of Computer and Telecommunication Systems IEEE 2001.

2. Ernst Biersack, Werner Geyer, "Synchronization Delivery and Play-out of Distributed Stored Multimedia Streams", Multimedia Systems, Vol. 7 N.1 , 70-90, 1999.

3. D. K. K. Y. Yau, S. S. Lam, "Adaptive Rate-Controlled Scheduling for Multimedia Applications", Multimedia 96 Processing, 4th ACM, Boston. Ma. pp. 129-140. 1996.

4. T. D. C. Little, and Arif Ghafoor, "Multimedia Synchronization Protocols for Broadband Integrated Services," IEEE Journal on selected Areas in Comm., Vol. 9, No.9, Dec. 1991.

5. Z. Chen, S. Than, R. Campbell, and Y.Li, "Real time video and audio in the world wide web", Proc. Fourth International World Wide Web Conference, 1995.

6. W. Geyer, "Stream Synchronization in a Scalable Video Server Array," Master's thesis, Institute Eurecom, Sophia Antipolis, France, Sept., 1995.

7. Dae-Jea Cho, Kee-Young Yoo, "The Study on Development of a Multimedia Synchronization Algorithm for Internet Based VOD Services", KIPS, Vol. 8-B, No 1, pp 74-80, Feb., 2001.

8. Gi-Sung Lee, Jeung-gyu Jee, Sok-Pal Cho, "Buffering Management Scheme for Multimedia Synchronization in Mobile Information System," Lecture Notes in Computer Science Vol. 2660, pp 545-554, June, 2003. 